\title{
BAK-SNEPPEN MODELS FOR THE EVOLUTION OF STRUCTURED KNOWLEDGE
}

\author{
Livio Clemente Piccinini \\ Ting Fa Margherita Chang \\ Maria Antonietta Lepellere \\ Mario Taverna \\ Giovanni Tubaro \\ DPIA, University of Udine, Italy
}

\begin{abstract}
Scientific knowledge is subject to a twin evolution, since its development towards novelty creates disconnections and inconsistencies, while the need of structure requires order and method so that transmission and comprehension can be ensured. Models of biological evolution can help to understand many social and economical phenomena where the search for optimality is hindered by voluntary or random competition. Bak-Sneppen is one of the most significant models because it balances at best explication power and simplicity. Unlike cellular automata models, Bak-Sneppen models join locality and globality. The authors try to re-read these models in the framework of mathematics, where, despite its high developped structure, knowledge waves can hinder comprehension both of pupils and of scholars. Some paradoxes are shown, where a greater knowledge diminishes efficiency and capability.
\end{abstract}

Keywords: Structured knowledge, Ontogeny versus Phylogeny, Mathematical teaching, BakSneppen Model, Partitioned Frames and Evolution.

\section{Introduction}

Instruction and consequent acquisition of a structured knowledge always were torn between a frame of imitation of examples (more or less exhaustive) and a frame of theoretical knowledge, exposed more or less systematically. Better reactions are usually achieved by imitation, while a stronger structure is acquired when a solid systematic basis is available, so that examples and experiments can be inserted in a solid frame. Theoretic basis alone is not sufficient, since it is difficult to be memorized and transferred into practice, while every knowledge requires to be recalled and strengthened.

Since the most ancient times mathematics and linguistic (as rhetoric) were subjects of methodological reflection. Many teaching experiences were attempted and descriptions are available. Mathematics found a conceptual foundation, challenging but transmissible, starting from Euclides' Elements 
(third century b.C.). They rapidly succeeded in acquiring a leading role as a fundamental reference and kept this role for over 1500 years. On the other side, linguistic found its basis in Panini's book (fourth century b.C) for Sanskrit, and in Cicero's and Quintilianus' works (first century b.C. and first century a.C.) for Latin, hence for a long teaching tradition in European cultural development. In this short note we refer to some moments of mathematics development.

We are dealing with the following problem: what happens when some part of didactics seems not to be "sufficient", and therefore must be modified? In the long period (a biologist should speak of Philogeny) this happens when new knowledge arises, as it was with de l'Hopital's fundamental book of 1696, where for the first time some techniques of differential calculus were made explicit, so that Newton's and Leibniz's researches could become a general purpose tool. Another critical step for mathematical analysis was the long evolution of the concept of limit, what still allows different didactical choices: should the limit of sequences be introducted first, or should the limit of functions be given at once, and should Euclidean space be used or a general topological space? Another problem is given by the fact that the definition of limit does not give any hint to the way how that is to be found.

A long lasting problem was a correct general definition of integral and nowadays it is to find the best way of introducing the theory to the students of various specializations (including alsdo pure mathematicians). On a more general level there is the secular difference between anglosaxon and continental approach: the first starts from the observation of physical phenomena, while the second starts from the concept of function and seems to lie inside mathematics, continuing the famous querelle between Newton and Leibniz. Incidentally we can recall that Italian students seem to be confused if they start from an anglosaxon text, because they have a good training in mathematics, but no particular training in physics.

In the short period of study of a young pupil (here we can speak of Ontogeny) on the contrary the continuous superimposition of new adjustments over previous knowledges (right or wrong does not matter) creates very often a conflict that confounds student's ideas.

The authors chose to insert this problems in a frame of interconnected selective evolution. A simple essential model seems to be Bak-Sneppen model, even if it is not suitable for quantitative statistical analysis, but only to express tendency trends.

The first section summarizes the model and some interesting cases studied by the authors, namely it is recalled how sectional evolution hinders the destruction of achieved objectives, but can also prevent further improvements (turris eburnea, ivory tower effect). 
The second section discusses some general behaviors that often happen in social sciences, and also in economics. It can be remarked that the acritic acceptance of models otherwhere successful happens also in education, with results that can lead to opposite effects.

The third section describes a case of mathematical long period phylogeny, namely two completely different approaches to the computation of a root, and shows how the method used in computers rather follows the fashion than a true search of efficiency.

Finally the fourth section describes an experiment performed at the University of Udine, where different behaviors in mathematical phylogeny can be recognized. They seem to insert Kanisza's conceptual frame into BakSneppen evolution model, allowing an interpretation connected with Freudenthal theory of sudden conceptual jumps.

\section{Inside Bak-Sneppen models}

The Bak-Sneppen model was originally introduced as an archetypical (or ideal) model of evolution by Per Bak and Kim Sneppen (Bak \& Sneppen, 1993) (compare also (Bak, 1996), (Piccinini et al., 2013)). After a comparison of many existing models that could explain (well) only some particular features of the phaenomena, ignoring many collateral aspects, they attained a synthetic model, that glues globality of information and locality of performance, but is not restricted to single cells. Their classical model, that has been developed in many more sophisticated forms, was defined as follows. There is a circle of $\mathrm{N}$ nodes and they are occupied by $\mathrm{N}$ different species, each of which has at present a random fitness. The fitness values are independent and uniformly distributed on $(0 ; 1)$. At each discrete time step the system is updated by locating the species with lowest fitness and replacing its fitness by a new random variable (between 0 and 1). This would in itself reach reach optimization of all the species. This is the utopian dream of instruction, social support, church and many other charitable institutions. The absence of result is usually justified by the lack of economical resources. But actually Bak-Sneppen model gives a more subtle explanation: when a species changes its fitness this action perturbs the two nearest species, that in turn change their own fitness at random, even if it were already good. Therefore while usually the worst species improves, the neighbors are turned at hazard. Bak-Sneppen models can be defined on a wide range of graphs using the same update rule as above. The model shows that this random processes can result in self-organization to a critical state. Threshold of fitness rises, rapidly at first, then exponentially slows until it reaches, around 0.66 , the critical state from which level extinction sweep back and forth through 
the ecosystem. Hence a general progress is anyhow achieved, since it becomes rare that a species falls under the critical level, while the distribution of values above is tendentially uniform.

Brunk in (Brunk, 2000) suggests that self-organized criticality is the sort of process that should have great intuitive appeal to social scientists. A large bibliography of applications of self organized criticality can be found in (Turcotte, 1999) (see also (Ausloos et al., 2004) for an economic application). The authors anyhow think that a sound basis for applying Bak-Sneppen model of contact with neighbors is given by Duesenberry's demonstration effect. Its first presentation can be found in (Duesenberry, 1949), while many application to social sciences can be found for example in (Cavalli, 1998).

Although the Bak-Sneppen model is extremely simple, it has not yet been completely solved in spite of numerous analytical and numerical investigations. A recent explicit solving formula for the case of 4 nodes (the smallest non obvious dimension) is to be found in (Schlemm, 2012). Motivated by the difficulty of analyzing rigorously even the one-dimensional version of the BakSneppen model, (Barbay \& Kenyon, 2001) proposed a simpler model with discrete fitness values. In their model each species has fitness 0 or 1 , and each new fitness is drawn from the Bernoulli distribution with parameter $p$. Since there are typically several least fit species, the process then repeatedly chooses at random a species for mutation among the least fit species. In case of small number of nodes explicit solutions can be found using Markov chains, as it was done by the authors in (Piccinini et al., 2013). Parameter $p$ can substitute up to a certain level a plurality of values, but it cannot explain the staircase phenomenon found by the authors in (Piccinini et al., 2013) and (Piccinini et al., 2014), and in particular the phaenomenon of overtaking in partitioned frames. Hence binary structure, though simple and appealing is not sufficient for a thorough description of what may happen. Anyhow also this case is by no means trivial, as it was shown by (Meester \& Znamenski, 2002).

Changes in the structure of the contacts have been studied (also in (Piccinini et al., 2013), table 5). In particular the classical Bak-Sneppen bilateral adjacent contact structure is somewhat more stable than other more erratic structures since random and scattered perturbations keep the average level of fitness at a lower level. This depends on the fact that in the classical model a long sequence of high values can be attacked only from its boundaries, so that it takes a long time before it undergoes a change (usually unfavourable). The natural step was to investigate systems where this structure becomes stable, what was performed in (Piccinini et al., 2014), section 3. There the global system is split in two or more subsystems that do not have contacts with each other so that only the subsystem where a minimum is found is subject to 
evolution, while all the other subsystems are preserved from any change. This protection increases the average fitness of the global system, both during transitions and in the terminal states. Remark that the only stable terminal states are those in which the cells of all the subsystems but one have reached the top level of fitness. It may take a very long number of iterations (infinite if the fitness is continuous) to reach this terminal situations, and usually some form of staircase is experimentally found, so that the average fitness seems to stay for a long period and then suddendly jumps to a new higher level (Staircase behavior described in the conclusions of (Piccinini et al., 2014)). When the number of fitness levels is greater than 2 a very important phenomenon may arise, namely "overtaking", as was explained in section 5, fig. 1, of (Piccinini et al., 2013): if the local minimum of a subsystem is higher than the global minimum, but some of its subjects have not reached the maximum, this system cannot change, so that it defends its good state, but it cannot improve. On the contrary for a subsystem where an absolute minimum exists there is the possibility not only of reaching but even of overtaking the "good" system, even if the probability is very low. This fact explains some sudden scientific, cultural, economic and political phenomena of overtaking: the principle "quieta non movere" (Let quiet things stay) may lead to standstill, in the models just like very often in real life.

\section{Construction and deconstruction of structured knowledge}

The basic Bak-Sneppen model asks a dramatic question: why those who are involved in the process of improving the weaker species may become worse? A first answer is obvious, because someone has to bear the costs, but the aforementioned Duesenberry model provides a further explanation. It is clarified by the brutal paradox of (Pfeffer \& Sutton, 2006) which is reported here:

Suppose you went to a doctor who said "I am going to do an appendicectomy on you"When you asked why, the doctor answered, "because I did one on my last patient and it made him better". We suspect you would hightail it out of the office, because you know that the treatment ought to fit the disease, regardless of whether or not the treatment helped the previous patient. Strangely enough, that logical thought happens more than we might care to admit in most companies.

We took this paradox from the book of (Brischi \& Marzano, 2015), where the uncritical imitation of a successful process is stigmatized. In economics the presentation of case studies leads ipso facto to consider them as best practices to follow. The spread of this theory is dominant, and, depending on the model of 
Bak-Sneppen, should lead to an overall improvement of the general situation, but the price is to admit ups and downs around the average level, what can be disappointing.

The tendency towards the imitation of the neighbor (real or virtual) often acts in the social sciences and economics, leading to differentiation, which is often masked as innovation. Broadening main fields of action in economy can be studied in the context of INPUT-OUTPUT matrices, as was done by (Droli et al., 2014) and by (Chang et al., 2015). The trend towards multi-functionality can also be recognized in the coalescence of territorial structures, as was shown by (Taverna et al., 2013).

Multifunctionality has dispersive character and often ends up creating a short period of damage rather than of benefit, as Bak-Sneppen model foresees. This is in agreement with the classic vision already mentioned by Dante in his Comedy (Alighieri, 2001)

“...Follow my steps, though all such wisper of you:

be as a tower of stone, its lofty crown

unswayed by anything the winds may do.

For when a man lets his attention range

toward every wisp, he loses true direction,

sapping his mind's force with continual change." Purg. 5, 13-18

“....it is no learning

to understand what one does not retain...." $\quad$ Par. 5, 41-42

In the last verses it is already covered the need to anchor the new acquisitions, without dispersion, as is well known by experts in advertising.

Two theoretical grounds ideal for a test are language teaching and especially teaching and research in mathematics, as it can be protected from external influences, although there are educational trends that seek to expand their scope of action. Here we meet the eternal competition between the core of a knowledge system and its additions, as described by the authors in (Piccinini et al., 2015) in the examples of the third section, in particular in formula (28).

The model of overtaking, mentioned in the previous section, seems to validate the traditional teaching techniques of separation between different areas, avoiding bundles of heterogeneous notions, the effectiveness of which may be of short duration.

The networks now allow to combine multi-functionality with specialization, though the cost is that of a virtual intermediation chain, composed either by human operators (typically poorly paid), or by automatic 
operators consisting of expert systems suitable to filter the information through the maze of networks see (MacCormick, 2012). The danger is that expert systems that learn by studying the behavior of users end up with the polarization into information hubs ignoring developments useful but not fashionable.

\section{Mathematical Phylogeny}

Originally, there are natural numbers and the language that expresses them. An extensive analysis of the inconsistencies and linguistic connotations that are associated with numbers was made in (Piccinini et al., 2015), with reference to (Eco, 2003), chap. 5 where he studied the loss and compensation. Mathematical philology, like all artistic and constructive activities, enters in the conceptual scheme of (Kubler, 1972), especially in chap. 3 dedicated to disposal and retention. It is interesting the dual role of the mathematician. On the one hand he is a builder who discards what is no longer useful, and on the other is a philologist / archaeologist / artist, who digs up and ennobles the past. The historical co-existence of the two roles will be shown by the example of the algorithms for the calculation of the square root.

Note that even an algebraic number as ,root of 2" from the operational point of view is known only as an approximation represented by the decimal sequence $1.41421 \ldots$.

The fact that it represents the diagonal of the unit square convinces one of its life, overcoming the Pythagorean problem of its non-commensurability with the side of the square, but does not make it operationally correct, as there is no appropriate tool to measure fractions over the second or third digit. The algorithms in use are all of the iterative type. What was once taught in school, despite its complexity due to the need to avoid repetition of calculations, was based on the introduction at each new step of the highest decimal digit without exceeding the value of 2 . On the other hand the computer uses a method that is inspired by the ancient approximation technique used by the Babylonians. The procedure is based on a geometric reasoning followed by a process of error compensation, both very natural. You are required to build a rectangle of fixed area $\mathrm{A}$ so that the base and height are equal. As an initial assumption suppose base is $b_{1}$. In correspondence the height is $h_{1}=A / b_{1}$. Excluding the fortunate case in which $b_{1}=h_{1}$ you must improve the choice, taking the base to an intermediate value between the old base and the old height, such as the average of the two, and the process is repeated. The formula becomes

$$
\mathrm{b}_{\mathrm{n}+1}=1 / 2\left(\mathrm{~h}_{\mathrm{n}}+\mathrm{b}_{\mathrm{n}}\right)=1 / 2\left(\mathrm{~A} / \mathrm{b}_{\mathrm{n}}+\mathrm{b}_{\mathrm{n}}\right)
$$


In the case of $\mathrm{A}=2$, starting from the initial value 1 the first terms are 1 , $1.5, \ldots 1.1417,1.41422 \ldots$. It is important to note that any initial positive value leads to a sequence that converges to the same limit $\sqrt{ } 2$, while starting from a negative value the sequence tends to $-\sqrt{ } 2$.

This raises several questions; why this method, old and quite intuitive, is not normally taught in primary schools? Nowadays every micro-computer calculates the square root so that manual calculation becomes unnecessary, but it was not taught even fifty years ago when an automatic instrument was not available. The reasons are at least three: it is necessary to have knowledge of the real numbers (not only as geometric intuition), the concept of limit of a sequence is required, some statistical idea is needed for stating that the average of two numbers gives an error minor of the difference between the two original numbers. Finally it should be proved that the sequence (1) defined by recurrence actually converges to a finite limit, and this limit is the very root of A. The continuity, even at the level of intuition, allows to understand that the limit is given by a rectangle in which the base and height are equal. The first course in mathematical analysis provides an initial response in the case of the equation (1) but a deepening and a generalization to a different choice of average (which is what is actually used in computers) requires the concept of contraction. Furthermore, since the process is stopped after a finite (and small) number of iterations, it is important to be able to estimate the residual error. From this point of view, the school system of computing one decimal digit after the other has the advantage of providing immediately a maximum for the error, and therefore it appears safer. In the case of equation (1), on the contrary, the estimation is not evident, as the sequence $b_{n}$ is a monotone decreasing sequence (starting from the second term), that therefore converges, but does not supply an immediate estimate of the error. The sequences that provide an easy estimation of the error are those nested obtained starting from a positive sequence that decreases towards 0 , in which then the terms are taken to alternating signs. Finally a constant, which will be the limit, is added.

$$
\mathrm{x}_{\mathrm{n}+1}<\mathrm{x}_{\mathrm{n}} \quad \mathrm{a}_{\mathrm{n}}=(-1)^{\mathrm{n}} \mathrm{x}_{\mathrm{n}} \quad \mathrm{b}_{\mathrm{n}}=\mathrm{L}+\mathrm{a}_{\mathrm{n}}
$$

In this case the error $\left|L-b_{n}\right|$ is estimated by the difference of the two consecutive terms $\left|b_{n-1}-b_{n}\right|$. In our case equation (1) is replaced by

$$
b_{n+1}=k N / b_{n}+(1-k) b_{n}
$$

If $1 / 2<\mathrm{k}<1$, then the sequence is nested, but the convergence to the limit becomes slower as $k$ approaches 1 . Therefore in the computer $k$ is chosen as a 
value simple to be represented in the binary system but not far from $1 / 2$. It was also noted that in binary system the school algorithm becomes extremely simple and the running time of the machine is less than that required by the elegant equation (3).

One explanation may be sought in the fact that in the initial years of computers the theory of contractions was very popular and was considered an advanced tool that scientifically characterized its users. It may also be noted that for the roots of higher order algorithms of type (1) are easy to use and to check. The basic algorithm for h-th root becomes

$$
\mathrm{x}_{\mathrm{n}+1}=\left[(\mathrm{h}-1) \mathrm{N} / \mathrm{x}_{\mathrm{n}}{ }^{\mathrm{h}-1}+\mathrm{x}_{\mathrm{n}}\right] / \mathrm{h}
$$

However, in view of the low frequency of use, it is in general reduced to the indirect calculation of $x^{y}$ with $y=1 / h$.

\section{Mathematical Ontogeny}

In mathematics the structured knowledge taught in the courses often destroys the ability to solve problems by intuition. Most general methods are learned theoretically and are not compared with the simpler, albeit less general, techniques. One obvious case is solving a quadratic equation in which, if there is suspicion that the solution is integer, it is easier to inspect directly the divisors of the known term, switching to formula only in case of failure. Experience shows that about half of the students of scientific courses ignore this shortcut, while conversely almost every person at the moment who has to solve the same problem as a game uses the simplified method, perhaps because the perception that the solution must really be integer is greater.

Vicario (Vicario, 1984) recalled an experiment of Kanisza showing that the increase in theoretical information reduced the ability to solve actual problems (for details see (Piccinini et al., 2015), note 12). Strictly related is the ontogeny of the interesting case of drawing a triangle knowing the three sides. The reader should try himself before reading what follows!

Use white paper (without lines and squares) and draw a triangle with three sides of length 4, 5 and 6. The only tool allowed is just a ruler, but neither compasses nor square.

It is possible that you decide to use analytic geometry (if you remember it) by solving an algebraic system like

$$
x^{2}+y^{2}=25 \quad(x-6)^{2}+y^{2}=16
$$


that is, $\mathrm{x}=15 / 4=3.75, \mathrm{y}=5 \sqrt{7} / 4 \approx 3.307$, for which a choice of possible vertices becomes (in a suitable Cartesian coordinate system) the triad $(0,0)$, $(6,0)$ and $(x, y)$. The available instrument is not ideal for working with the Cartesian coordinates, especially in the absence of an orthogonal network, however, a solution more or less approximate can be found. How can you verify and improve it? But it could be established that the tool is a piece of white paper where the three lengths $(4,5,6)$ are marked on the margin and nothing else. The unavailability of a metric system prevents you from using the solution of the algebraic system (5). What to do?

We conducted the experiment with two different cohorts of students at the University of Udine. The cohort of engineering students (first year) knew in its totality (possibly through the suggestion of neighbors) the classical Euclidean construction, made with ruler and compass. Once fixed the first two points at a distance (e.g.) 6 the probem was thus to approximate the construction of an arc of a circle of radius 5 centered at the first point using the segment on the sheet of lenght 5 and rotating, thus obtaining a simulacrum of the circle. Similarly for the other circle. Here too, the question is the same: how to verify and improve the result? At this point the construction of Euclid, without compass, becomes an unnecessary complexity. The other cohort was formed by students (mostly girls) of Educational Science (first year). Almost all students presented a very efficient solution, suitable also to be taught in primary school. From the first point (A) draw a segment of length (e.g.) 5 with a random angle, obtaining a provisional point $\mathrm{C} 1$. From $\mathrm{B}$ trace the ray that passes through $\mathrm{C} 1$ and mark the point $\mathrm{C} 2$ at distance 4 from point $\mathrm{B}$. In general $\mathrm{C} 2$ does not coincide with the point $\mathrm{C} 1$, otherwise the problem is solved. Draw now the half-line from $\mathrm{A}$ passing through the point $\mathrm{C} 2$ and draw the point at distance 5 from $\mathrm{A}$, and so on, alternating the starting points $\mathrm{A}$ and $\mathrm{B}$. After two or three iterations it happens that the points are stabilized, and this is a solution (approximate) of the problem. It is therefore clearly a convergent iterative method (though the students did not have the mathematical knowledge to know this fact).

The iterative formula, wanting to simulate the process on the computer is as follows. Denote by $a$ the distance $\mathrm{AB}, b$ the distance AC, $c$ the distance $\mathrm{BC}$; $\vartheta$ be angle BAC, $\phi$ the angle ABC. Start from an arbitrary angle $\vartheta_{1}$. The recurrence is

$$
\phi_{\mathrm{k}}=\arctan \left[\mathrm{b} \sin \vartheta_{\mathrm{k}} /\left(-\mathrm{a}+\mathrm{b} \cos \vartheta_{\mathrm{k}}\right)\right], \vartheta_{\mathrm{k}+1}=\arctan \left[\mathrm{c} \sin \phi_{\mathrm{k}} /\left(\mathrm{a}+\mathrm{c} \cos \phi_{\mathrm{k}}\right)\right] .
$$

The confrontation between students of the two cohorts lead engineers to understand that their trial and error process of drawing points of the circles becomes selected and streamlined through the process of students of education 
sciences. Two facts can be appreciated: rigorous seclusion prevents improvements and allows overtaking; in the learning of mathematics progress is tied with sudden discontinuities as fully described by (Freudenthal, 1991) with some examples starting from (Freudenthal, 1975).

\section{Conclusions}

The structured knowledge acts both at the level of phylogeny, since it settles over time and builds a system of consistent relations, and in the ontogeny of the individual acquisition. The moments of confrontation between different structures or innovation sparks need time to affect the existing structures. The history of philosophy first and of science later are full of secular changes, and this happened in mathematics as well, even if it is less susceptible of perturbation. The Thucydidean $\kappa \tau \eta \mu \alpha \varepsilon \sigma \alpha 1 \varepsilon 1$ (purchase forever, I, 22) holds, but still there are secular variations, especially in individual ontogeny according to Freudenthal in (Freudenthal, 1983). Two sections of this paper are devoted to mathematics, with examples simple enough to be understood also by laics (the authors hope so). The Bak-Sneppen evolution model of species is a valuable starting point for the interpretation of these phenomena.

The linguistic area is another field where the model operates as phylogeny, (especially phenomena of contact). At the level of individual ontogeny, in glottodidactic, structured grammars and systematic thesaurus meet and sometimes clash with methods based on practice and assimilation of microsystems. The authors touched in (Piccinini et al., 2015) a small stretch of encounter between mathematics and linguistics, but the experiment of comparing the two subjects deserves to be resumed and expanded, keeping in mind the work of (Petit-Cocorda, 1985).

\section{References}

Alighieri, D. (2001). The Divine Comedy: The Inferno, The Purgatorio, and The Paradiso. Translated by Ciardi, J. New York: Signet Classics.

Ausloos, M., Clippe, P., \& Pekalski, A. (2004). Evolution of economic entities under heterogeneous political/environmental conditions within a Bak-Sneppen-like dynamics. Physica A: Statistical Mechanics and its Applications, 332, 394-402.

Bak, P. (1996). How Nature Works: The Science of Self-Organized Criticality. New York: Springer-Verlag.

Bak, P., \& Sneppen, K. (1993). Punctuated equilibrium and criticality in a simple model of evolution. Physical Review Letters. 71 (24), 4083-4086.

Barbay, J., \& Kenyon, C. (2001). On the discrete Bak-Sneppen model of self-organized criticality. Proceeding of the Twelfth Annual ACM-SIAM Symposium on Discrete Algorithms (pp. 928-934). Washington, DC. 
Livio Clemente Piccinini, Ting Fa Margherita Chang, Maria Antonietta Lepellere, Mario

Taverna, Giovanni Tubaro. Bak-Sneppen Models for the Evolution of Structured Knowledge

Brischi, S., \& Marzano, G. (2015). Management e Rottamazione. Milano-Udine: Mimesis, 1920.

Brunk, G. G. (2000). Understanding Self-Organized Criticality as a Statistical Process. John Wiley \& Sons, Inc., 5 (3), 26-33.

Cavalli, A. (1998). Sociologia, Enciclopedia delle Scienze Sociali, VIII. Roma: Treccani.

Chang, T. F. M., Iseppi, L., \& Droli, M. (2015). Extra-core production and capabilities: where is the Food Industry going?. International Food and Agribusiness Management Review, 18, 105-126.

Droli, M., Chang, T. F. M., Iseppi, L., \& Piccinini, L. C. (2014). Managing trade contacts in HotRest intermediate markets: a resource-based view analysis in EU countries. Tourism Economics, 20, 757-778.

Duesenberry, J. S. (1949). Income, Savings and Theory of Consumer Behaviour. Cambridge Massachusetts: Harvard University Press, 89-125.

Eco, U. (2003). Dire quasi la stessa cosa. Esperienze di traduzione. Milano: Bompiani, 95138.

Freudenthal, H. (1975). Wandelingen met Bastiaan. Pedomorfose, 25, 51-64.

Freudenthal, H. (1983). Wie entwickelt sich reflexives Denken? Neue Sammlung, 23, 485-497.

Freudenthal, H. (1991). Revisiting Mathematical Education. China Lectures. Dordrecht: Kluwer Academic Publishers. Sect. 2.3.3.

Kubler, G. (1972). The Shape of Time. New Haven: Yale University Press.

MacCormick, J. (2012). Nine Algorithms that Changed the Future. Princeton: Princeton University Press, Chapters 2 and 3.

Meester, R., \& Znamenski, D. (2002). Non-triviality of a discrete Bak-Sneppen evolution model. Journal of Statistical Physics, 109, 987-1004.

Petit-Cocorda, J. (1985). Morphogenèse du Sens. Paris: Presses Universitaires de France, Part. Chapter 5.

Pfeffer, J., \& Sutton, R. (2006). Hard Facts, Dangerous Half-Truths, and Total Nonsense. USA: Harvard Business School Press, p. 8.

Piccinini, L. C., Lepellere, M. A., \& Chang, T. F. M. (2013). Utopias of Perfection and their dystopias. SOCIETY, INTEGRATION, EDUCATION. Proceedings of the International Scientific Conference, v. 3, p. 189-200, june. 2013. ISSN 1691-5887.

Piccinini, L. C., Lepellere, M. A., Chang, T. F. M., \& Iseppi, L. (2014). Partitioned Frames in Bak Sneppen Models. Italian Journal of Pure and Applied Mathematics, 33, 461-488.

Piccinini, L. C., Taverna, M., Chang, T. F. M., \& Tubaro, G. (2015). Perception, Connotation, Translation of Numbers. SOCIETY, INTEGRATION, EDUCATION. Proceedings of the International Scientific Conference, v. 3, p. 183-192, may. 2015. ISSN 1691-5887.

Piccinini, L. C., Lepellere, M. A., Chang, T. F. M., \& Iseppi, L. (2015). Endogenous Control in a Ternary Lotka-Volterra Model and its Applications. Italian Journal of Pure and Applied Mathematics, 35, 677-704.

Schlemm, E. (2012). Asymptotic Fitness Distribution in the Bak-Sneppen Model of Biological Evolution with four Species. Journal of Statistical Physics, 148, 191-203.

Taverna, M., Piccinini, L. C., Chang, T. F. M., \& Iseppi, L. (2013). Structures and Paths for the Exploration of Landscape-Cultural Mosaic. SOCIETY, INTEGRATION, EDUCATION. Proceedings of the International Scientific Conference, v. 1, p. 517-527, may. 2013. ISSN 1691-5887. 

Proceedings of the International Scientific Conference. Volume IV, May $27^{\text {th }}-28^{\text {th }}$, 2016. 109-121

Turcotte, D. L. (1999). Self-organized criticality. Reports on Progress in Physics, 62, 13771429.

Vicario, G. B. (1984). Private communication to L.C. Piccinini. Udine: Department of Mathematics. 